\title{
CARACTERÍSTICAS SOCIODEMOGRÁFICAS DE MUJERES PERUANAS CON VIRUS PAPILOMA HUMANO DETECTADO POR PCR-RFLP
}

\author{
Yasser Sullcahuaman-Allende ${ }^{1, a}$, María del Carmen Castro-Mujica',a, Roberto Mejía Farro ${ }^{1, b}$, \\ Carlos A. Castaneda ${ }^{1, \mathrm{c}}$, Miluska Castillo ${ }^{1, \mathrm{~d}}$, Ketty Dolores-Cerna ${ }^{1, \mathrm{e}}$, Ebert Poquioma $^{1, \mathrm{f}}$
}

\begin{abstract}
RESUMEN
Con el objetivo de determinar las características sociodemográficas del virus de pacientes con papiloma humano (VPH) referidas al Instituto Nacional de Enfermedades Neoplásicas (INEN) durante los años 2012-2014, se realizó la detección del VPH en células cervicales por reacción en cadena de la polimerasa (PCR). En 465 muestras cervicales se detectaron $151(32,5 \%)$ casos de VPH positivas. Los genotipos más frecuentes fueron VPH-16 (23,8\%) y VPH-6 (11,9\%). La presencia de VPH fue mayor en mujeres de 17 a 29 años (OR 2,64, IC 95\%:1,14-6,13) y solteras (OR 2,31, IC 95\%: 1,37-3,91), la presencia de genotipos de VPH de alto riesgo fue mayor en solteras (OR 2,19, IC 95\%: 1,04-4,62). En conclusión, mujeres jóvenes y solteras presentaron mayor frecuencia de casos VPH-positivos a quienes se debe enfatizar la participación en programas de tamizaje con métodos moleculares y citológicos combinados, a fin de detectar oportunamente el riesgo de desarrollar cáncer de cuello uterino.
\end{abstract}

Palabras clave: Papiloma virus humano; Genotipo; Reacción en cadena de la polimerasa (fuente: DeCS BIREME).

\section{DEMOGRAPHIC CHARACTERISTICS OF HUMAN PAPILLOMAVIRUS DETECTED BY PCR-RFLP IN PERUVIAN WOMEN}

\begin{abstract}
In order to determine the sociodemographic characteristics of human papillomavirus (HPV) in patients referred to the National Institute of Neoplastic Diseases (INEN) between 2012-2014, the detection of HPV in cervical cells was performed by polymerase chain reaction (PCR). In 465 cervical samples, 151 (32.5\%) cases were HPV positive. The most common genotypes were HPV-16 (23.8\%) and HPV-6 (11.9\%). The presence of HPV was higher in women aged 17-29 years (OR $=2.64,95 \% \mathrm{Cl} 1.14$ to 6.13$)$ and single women $(\mathrm{OR}=2.31,95 \% \mathrm{Cl} 1.37$ to 3.91$)$. The presence of genotypes of high-risk HPV was higher in single women $(\mathrm{OR}=2.19,95 \% \mathrm{Cl} 1.04$ to 4.62$)$. In conclusion, young and single women had a higher frequency of HPV-positive cases. Therefore participation by these groups should be emphasized in screening programs with combined molecular and cytological methods in order to detect the risk of developing cervical cancer in a timely manner.
\end{abstract}

Key words: Human papilloma virus; Genotype; Polymerase chain reaction (source: MeSH NLM).

\section{INTRODUCCIÓN}

El cáncer de cuello uterino (CCU) es la neoplasia más frecuente y mortal en la población femenina peruana, presentándose aproximadamente 4600 casos nuevos por año y provocando cerca de 1700 muertes al año ${ }^{(1)}$. La infección persistente por los genotipos oncogénicos virus de papiloma humano (VPH) es un agente causal responsable del desarrollo del CCU. EI ADN de VPH se encuentra presente hasta en $98 \%$ de los CCU invasivos ${ }^{(2,3)}$.
El VPH se clasifica en más de 189 genotipos según la secuencia de su ADN y 40 de estos son capaces de infectar el cuello uterino. Se subdividen en genotipos de bajo riesgo (BR) (VPH - 6, 11, 13, 40, 42, 43, 44, 54, $59,61,70,72,81)$ y alto riesgo (AR) (VPH - 16, 18, 31, $33,35,39,45,51,52,56$ y 58 ) según su probabilidad de producir CCU. Un reciente estudio con 8977 especímenes de CCU VPH positivo encontró que los genotipos de AR son responsables de alrededor del $90 \%$ de los casos, y los genotipos 16/18 son responsables del $70 \%$ de los casos en el mundo ${ }^{(4)}$. En forma similar,

Instituto Nacional de Enfermedades Neoplásicas. Lima, Perú.

Médico genetista; ${ }^{\mathrm{d}}$ biólogo molecular; ${ }^{\mathrm{c}}$ oncólogo clínico, magíster en Genética y Biología Celular, magíster en oncología avanzada; ${ }^{\mathrm{d}}$ toxicólogo; ${ }^{\mathrm{e}}$ máster en docencia en Investigación; ${ }^{\mathrm{f}}$ médico cirujano, magíster en Salud Pública.

Recibido: : 22-12-15 Aprobado: 17-06-15

Citar como: Sullcahuaman-Allende Y, Castro-Mujica MDC, Mejía Farro R, Castañeda CA, Castillo M, Ketty Dolores-Cerna K, Poquioma E. Características sociodemográficas de mujeres peruanas con virus papiloma humano detectado por PCR-RFLP. Rev Peru Med Exp Salud Publica. 2015;32(3):509-14. 
Santos et al. encuentran la presencia de DNA de PVH en más del $90 \%$ de los CCU en mujeres peruanas ${ }^{(5)}$.

Se han desarrollado diversos métodos de tamizaje de infección por VPH, sin embargo, el análisis molecular mediante reacción en cadena de polimerasa $(\mathrm{PCR})$ permite detectar hasta una molécula de DNA de VPH en $10^{5}$ células y es considerada la técnica más sensible de detección de

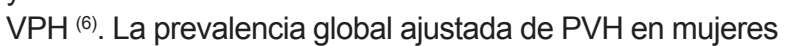
con citología normal es alrededor de $10 \%{ }^{(5,7,8)}$.

Factores sociodemográficos como la clase social han sido fuertemente asociados con el diagnóstico de CCU, sin embargo, la información es menor para los factores asociados con infección por VPH. La identificación de factores asociados a esta infección viral permitirá el diseño de estrategias de políticas de salud pública para reducir la mortalidad por $\operatorname{CCU}{ }^{(9,10)}$.

El propósito del presente estudio fue determinar la asociación entre la infección y genotipos de VPH en muestras cervicales evaluadas mediante el estudio por PCR y enzimas de restricción y factores sociodemográficos en mujeres peruanas referidas para tamizaje al Laboratorio de Biología Molecular del Instituto Nacional de Enfermedades Neoplásicas (INEN).

\section{EL ESTUDIO}

\section{MUESTRAS CERVICALES}

Entre los años 2011 a 2014 se recibieron 465 muestras de escobillados cervicales de pacientes sin diagnóstico de cáncer cervical remitidas de diversos centros de salud de la ciudad de Lima, al Laboratorio de Biología Molecular del INEN, para el estudio molecular y genotipificación de VPH se realizó la extracción del ADN de las muestras con el kit comercial Purelink Genomic DNA minikit Invitrogen por sistema de columnas, y la cuantificación por fluorometría, donde la mayoría de muestras tenía una concentración mayor a $100 \mathrm{ug} / \mathrm{mL}$.

\section{DETECCIÓN Y GENOTIPIFICACIÓN DEL VPH}

EI DNA de VPH fue extraído y almacenado a $-20{ }^{\circ} \mathrm{C}$. Fue analizado mediante PCR y de acuerdo con la metodología descrita previamente ${ }^{(11,12)}$. Se empleó el programa de amplificación Concensus Primers-PVH $\mathrm{Y}$ Gen betaglobina humana (tiempo aproximado $1 \mathrm{~h}$ $32 \mathrm{~min}$ ), usando las siguientes condiciones: $94{ }^{\circ} \mathrm{C}$ por 5 min; $94{ }^{\circ} \mathrm{C}$ por $30 \mathrm{~s} ; 56{ }^{\circ} \mathrm{C}$ por $35 \mathrm{~s} ; 68^{\circ} \mathrm{C}$ por 35 $\mathrm{s}$; $68^{\circ} \mathrm{C}$ por $5 \mathrm{~min}$, y por ultimo $4{ }^{\circ} \mathrm{C}$ indefinidamente, sumando 35 ciclos de amplificación. En resumen, se utilizaron cebadores específicos MY09/MY11 para el gen L1 de la región común de los VPH correspondiente a una banda de $450 \mathrm{pb}$. Posteriormente, en los casos de detección VPH positivos, se determinaron por PCRRFLP los genotipos VPH-6, 11, 16, 18, 31 y 33 mediante la acción de la enzima de restricción AFA1. Los casos positivos en los que no se pudo determinar el genotipo fueron catalogados como VPH-X ${ }^{(13)}$.

Como control de calidad de la extracción del ADN se utilizaron cebadores para el gen de la beta globina humana (PCO4-GH20) que corresponde a una banda de $268 \mathrm{pb}$. El control positivo fue una biopsia que corresponde al VPH tipo 16 de 310 pb y un blanco como control negativo, los productos amplificados fueron corridos en un sistema de electroforesis horizontal empleando gel de agarosa al 3\% a 112 voltios por 40 minutos que luego se coloreó durante 15 minutos con SYBR Safe y se visualizó en el transiluminador UV. Se tomaron las impresiones fotográficas y se editaron al formato de resultado de las pacientes.

\section{CLASIFICACIÓN}

Se clasificaron los genotipos de VPH en relación a su asociación con el cáncer de cuello uterino, resultando tres categorías: VPH de AR oncogénico (VPH: 16, 18, 31, 33), el VPH de BR oncogénico (VPH: 6 y 11) y el $\mathrm{VPH}$ de riesgo no definido (VPH-X) ${ }^{(2,3)}$.

\section{INFORMACIÓN EPIDEMIOLÓGICA}

Se obtuvo la información epidemiológica de las pacientes, incluidas en nuestra serie a través del sistema informático del Registro Nacional de Identificación y Estado Civil (RENIEC). La información obtenida en esta metodología fue fecha de nacimiento, dirección de domicilio, grado de instrucción y estado civil.

\section{ANÁLISIS ESTADÍSTICO}

Como el análisis de detección de los subtipos de VPH en muestras cervicales fue considerado positivo o negativo, empleamos regresión logística simple para establecer si las características epidemiológicas se asociaron a la infección de alto riesgo y total, estableciendo OR crudos con sus intervalos de confianza (IC) al 95\%. Luego, se estableció mediante regresión logística múltiple los OR ajustados con sus IC al 95\%. Se empleó el software estadístico Stata versión 12, considerando un nivel de significación de 0,05.

\section{CONSIDERACIONES ÉTICAS}

La información obtenida se mantuvo en completa confidencialidad. El proyecto del estudio fue aprobado por el Comité Revisor de Protocolo y el Comité de Ética del Instituto Nacional de Enfermedades Neoplásicas. 
Tabla 1. Resultado de VPH en muestras cervicales referidas al Instituto Nacional de Enfermedades Neoplásicas según características sociodemográficas

\begin{tabular}{|c|c|c|c|c|c|c|}
\hline & \multicolumn{2}{|c|}{ Total } & \multicolumn{2}{|c|}{ Positivo } & \multicolumn{2}{|c|}{ Alto riesgo } \\
\hline & $(n=465)$ & $(\%)$ & $(n=151)$ & $(\%)$ & $(n=67)$ & $(\%)$ \\
\hline Mediana de edad +/-DIQ & $33,5+/-7,5$ & & $32+/-6$ & & $31+/-6$ & \\
\hline \multicolumn{7}{|l|}{ Edad } \\
\hline $17-29$ & 162 & $(34,8)$ & 67 & $(44,4)$ & 30 & $(44,8)$ \\
\hline $30-39$ & 160 & $(34,4)$ & 48 & $(31,8)$ & 22 & $(32,8)$ \\
\hline $40-49$ & 104 & $(22,4)$ & 27 & $(17,9)$ & 11 & $(16,4)$ \\
\hline$\geq 50$ & 38 & $(8,2)$ & 8 & $(5,3)$ & 4 & $(6,0)$ \\
\hline Desconocido & 1 & $(0,2)$ & 1 & $(0,7)$ & 0 & $(0,0)$ \\
\hline \multicolumn{7}{|l|}{ Ubicación del distrito } \\
\hline Norte & 57 & $(12,3)$ & 14 & $(9,3)$ & 9 & $(13,4)$ \\
\hline Este & 87 & $(18,7)$ & 24 & $(15,9)$ & 14 & $(20,9)$ \\
\hline Centro & 142 & $(30,5)$ & 49 & $(32,5)$ & 21 & $(31,3)$ \\
\hline Sur & 16 & $(3,4)$ & 9 & $(6,0)$ & 3 & $(4,5)$ \\
\hline Callao & 25 & $(5,4)$ & 13 & $(8,6)$ & 2 & $(3,0)$ \\
\hline Desconocido & 138 & $(29,7)$ & 42 & $(27,8)$ & 18 & $(26,9)$ \\
\hline \multicolumn{7}{|l|}{ Estado civil } \\
\hline Soltera & 301 & $(64,7)$ & 112 & $(74,2)$ & 50 & $(74,6)$ \\
\hline Casada/Divorciada/Viuda & 108 & $(23,2)$ & 22 & $(14,6)$ & 9 & $(13,4)$ \\
\hline Desconocido & 56 & $(12,0)$ & 17 & $(11,3)$ & 8 & $(11,9)$ \\
\hline \multicolumn{7}{|l|}{ Grado de instrucción } \\
\hline Sin instrucción & 9 & $(1,9)$ & 5 & $(3,3)$ & 2 & $(3,0)$ \\
\hline Primaria & 49 & $(10,5)$ & 7 & $(4,6)$ & 4 & $(6,0)$ \\
\hline Secundaria & 306 & $(65,8)$ & 110 & $(72,8)$ & 49 & $(73,1)$ \\
\hline Superior/Técnico & 44 & $(9,5)$ & 11 & $(7,3)$ & 4 & $(6,0)$ \\
\hline Desconocido & 57 & $(12,3)$ & 18 & $(11,9)$ & 8 & $(11,9)$ \\
\hline
\end{tabular}

\section{RESULTADOS}

Un total de 465 mujeres fueron evaluadas con una mediana de edad de 33,5 $\pm 7,5$ años (rango de 17 a 78). La mayoría de los casos tenían domicilio en Lima $(67,1 \%)$. Según su procedencia distrital en Lima, el 12,7\% tenían domicilio en San Juan de Lurigancho; el 8,0\% en la Victoria y en Lima Cercado; el 6,2\% en San Martin de Porras y el $3,4 \%$ en Santiago de Surco. El grado de instrucción fue, en la mayoría, secundaria completa $(65,8 \%)$, seguido de primaria completa $(10,5 \%)$ y superior/técnico $(9,5 \%)$. El estado civil predominante fue soltera $(64,7 \%)$, seguido de casada $(20,2 \%)$ (Tabla 1$)$.

La detección del VPH fue positiva en 151 (32,5\%) casos. Se pudo identificar el genotipo en $93(61,6 \%)$ de los casos positivos. El 44,4\% de los casos positivos pertenecieron a VPH-AR y el 55,6\% a VPH-BR. Se encontraron los genotipos VPH-16 en $23,8 \%$; VPH-31 en $8,0 \%$; VPH-18 en $3,3 \%$; VPH-33 en $3,3 \%$; VPH-6 en $11,9 \%$, y VPH-11 en $5,3 \%$ de los casos positivos. El $6 \%$ de los casos positivos presentó dos genotipos de VPH simultáneamente (tres casos VPH 6 y VPH 16; tres casos VPH 16 y VPH 31; un caso VPH 11 y VPH 33, y un caso VPH 16 y VPH 33). Un caso presentó tres genotipos de VPH 6, 16 y 31; el cual se trató de una paciente de 24 años, soltera y con secundaria completa (Tabla 4), (Figura 1).

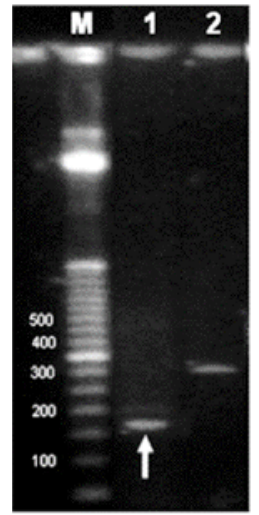

Caso VPH 6

M: Marcador de peso molecular 1: Paciente PVH-6 POSITIVO (159 pb) 2: Control PVH-16 POSITIVO (310 pb)

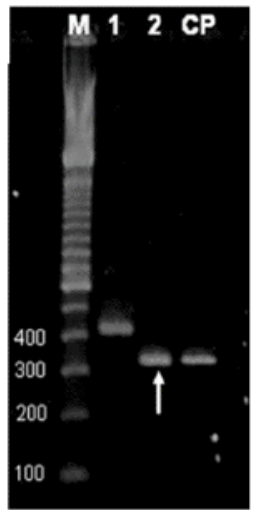

Caso VPH 16

M: Marcador de peso molecular 1: Paciente PVH-POSITIVO (450 pb) 2: Paciente PVH-16 POSITIVO (310 pb) 3: Control PVH-16 POSITIVO (310 pb)
Figura 1. Detección y genotipificación del VPH por PCRRFLP en muestras cervicales referidas al Instituto Nacional de Enfermedades Neoplásicas, 2012-2014

El modelo de regresión logística crudo encontró que la detección de ADN del VPH se relaciona directamente con edad joven (OR=2,64, IC 95\%: 1,14-6,13, $p=0,023)$ y estado civil soltera (OR=2,31, IC 95\%: 1,37-3,91, $p=0,002$ ) (Tabla 2). La posibilidad de detectar un genotipo de VPH-AR se asocia con el estado civil soltera (OR=2,19, IC 95\%: 1,04-4,62, $p=0,040)$ (Tabla 3). 
Tabla 2. Características sociodemográficas de los resultados de $\mathrm{VPH}$ en muestras cervicales referidas al Instituto Nacional de Enfermedades Neoplásicas

\begin{tabular}{|c|c|c|c|c|c|c|}
\hline & $\begin{array}{c}\text { OR } \\
\text { crudo }\end{array}$ & (IC 95\%) & Valor $p$ & $\begin{array}{c}\text { OR } \\
\text { ajustado }\end{array}$ & (IC 95\%) & $\begin{array}{c}\text { Valor } \\
p \\
\end{array}$ \\
\hline \multicolumn{7}{|l|}{ Edad } \\
\hline $17-29$ & 2,64 & $(1,14-6,13)$ & 0,023 & 1,80 & $(0,68-4,80)$ & 0,239 \\
\hline $30-39$ & 1,61 & $(0,69-3,76)$ & 0,274 & 1,40 & $(0,53-3,68)$ & 0,491 \\
\hline $40-49$ & 1,31 & $(0,54-3,22)$ & 0,549 & 1,12 & $(0,42-2,99)$ & 0,814 \\
\hline$\geq 50$ & 1,00 & & & 1,00 & & \\
\hline \multicolumn{7}{|c|}{ Grado de instrucción } \\
\hline $\begin{array}{l}\text { Sin ins- } \\
\text { trucción }\end{array}$ & 3,75 & $(0,85-16,5)$ & 0,080 & 3,72 & $(0,79-17,37)$ & 0,095 \\
\hline Primaria & 0,50 & $(0,17-1,43)$ & 0,196 & 0,43 & $(0,15-1,26)$ & 0,126 \\
\hline $\begin{array}{l}\text { Secun- } \\
\text { daria }\end{array}$ & 1,68 & $(0,82-3,46)$ & 0,157 & 1,27 & $(0,60-2,71)$ & 0,531 \\
\hline $\begin{array}{l}\text { Superior/ } \\
\text { Técnico }\end{array}$ & 1,00 & & & 1,00 & & \\
\hline $\begin{array}{l}\text { Estado } \\
\text { civil } \\
\text { soltera* }\end{array}$ & 2,31 & $(1,37-3,91)$ & 0,002 & 1,84 & $(1,05-3,21)$ & 0,032 \\
\hline
\end{tabular}

${ }^{*}$ Comparado con el grupo de casada/divorciada/viuda

En el análisis multivariado el estado civil soltera se asocia a la presencia de ADN del VPH (OR=1,84, IC 95\%: 1,05$3,21, p=0,032$ ) (Tabla 2), mientras que la presencia de genotipos de VPH-AR no presentó variables asociadas (Tabla 3).

\section{DISCUSIÓN}

El presente trabajo es el primero, a nuestro conocimiento, que describe la frecuencia de los genotipos del VPH en muestras cervicales y su asociación con características sociodemográficas en mujeres peruanas.

Tabla 3. Características sociodemográficas de los resultados de VPH de alto riesgo en muestras cervicales referidas al Instituto Nacional de Enfermedades Neoplásicas

\begin{tabular}{|c|c|c|c|c|c|c|}
\hline & $\begin{array}{c}\text { OR } \\
\text { crudo }\end{array}$ & (IC 95\%) & $\begin{array}{c}\text { Valor } \\
p\end{array}$ & $\begin{array}{c}\text { OR } \\
\text { ajustado }\end{array}$ & (IC 95\%) & $\begin{array}{c}\text { Valor } \\
p\end{array}$ \\
\hline \multicolumn{7}{|l|}{ Edad } \\
\hline $17-29$ & 1,93 & $(0,64-5,86)$ & 0,245 & 1,30 & $(0,37-4,62)$ & 0,682 \\
\hline $30-39$ & 1,36 & $(0,44-4,19)$ & 0,598 & 1,06 & $(0,30-3,74)$ & 0,923 \\
\hline $40-49$ & 1,01 & $(0,30-3,71)$ & 0,993 & 0,82 & $(0,23-2,97)$ & 0,765 \\
\hline$\geq 50$ & 1,00 & & & 1,00 & & \\
\hline \multicolumn{7}{|c|}{ Grado de instrucción } \\
\hline $\begin{array}{l}\text { Sin } \\
\text { instrucción }\end{array}$ & 2,86 & $(0,44-18,68)$ & 0,273 & 2,62 & $(0,37-18,69)$ & 0,337 \\
\hline Primaria & 0,89 & $(0,21-3,79)$ & 0,873 & 0,79 & $(0,18-3,43)$ & 0,756 \\
\hline Secundaria & 1,91 & $(0,65-5,57)$ & 0,238 & 1,50 & $(0,49-4,55)$ & 0,477 \\
\hline $\begin{array}{l}\text { Superior/ } \\
\text { Técnico }\end{array}$ & 1,00 & & & 1,00 & & \\
\hline $\begin{array}{l}\text { Estado civil } \\
\text { soltera** }\end{array}$ & 2,19 & $(1,04-4,62)$ & 0,040 & 1,84 & $(0,83-4,05)$ & 0,131 \\
\hline
\end{tabular}

${ }^{*}$ Comparado con el grupo de casada/divorciada/viuda
Tabla 4. Genotipos positivos de muestras cervicales referidas al Instituto Nacional de Enfermedades Neoplásicas, 2012-2014

\begin{tabular}{lcc}
\hline Genotipos & $\mathbf{n = 1 5 1}$ & $\mathbf{( \% )}$ \\
\hline Genotipos simples & & \\
VPH-16 & 36 & $(23,8)$ \\
VPH-6 & 18 & $(11,9)$ \\
VPH-31 & 12 & $(8,0)$ \\
VPH-11 & 8 & $(5,3)$ \\
VPH-18 & 5 & $(3,3)$ \\
VPH-33 & 5 & $(3,3)$ \\
Genotipos mixtos & & \\
VPH6-VPH16 & 3 & $(2,0)$ \\
VPH16-VPH31 & 3 & $(2,0)$ \\
VPH16-VPH33 & 1 & $(0,7)$ \\
VPH11-VPH33 & 1 & $(0,7)$ \\
VPH6-VPH16-VPH31 & 1 & $(0,7)$ \\
Genotipo VPH-X & 58 & $(38,4)$ \\
\hline
\end{tabular}

Se encontraron 151 casos (32,5\%) con presencia del ADN de VPH en nuestra serie de pacientes referidas de distintos centros. Esta frecuencia es mayor que la reportada por Santos et al. ${ }^{(5)}$ quien encuentra una frecuencia de infección cervical de $17,7 \%$ en su grupo control (196 mujeres sanas provenientes de la ciudad de Lima), y que la reportada por Almonte et al. quien encuentra una frecuencia de $12,6 \%$ en 5435 muestras cervicales de la red de salud del departamento de San Martin durante el $2001{ }^{(8)}$. Sin embargo, bastante similar al 34,5\% de infección por PVH de alto riesgo reportado por Iwasaki et al. en una serie de 2247 muestras cervicales de mujeres de áreas urbanas de diferentes departamentos del Perú obtenidas entre 2011 y

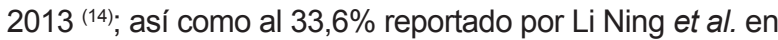
población de distintas ciudades de Perú ${ }^{(15)}$.

En relación a la prevalencia de otros países de la región, fue mayor que en Bolivia (3\%), Chile (14\%), México $(14,5 \%)$, Colombia $(14,8 \%)$ o Costa Rica $(16 \%)$. Sin embargo, es inferior a la reportada en adolescentes de Estados Unidos (64\%), en trabajadoras sexuales de Corea $(47 \%)$ o mujeres brasileras con lesiones cervicales $(43 \%)^{(16-20)}$. Esta diferencia puede deberse a que la selección de las pacientes incluidas en el presente tamizaje fue realizada por el médico tratante en la institución de donde proviene el caso. Así, estos casos podrían pertenecer a una población de mayor riesgo de infección que la población promedio.

La alta frecuencia de cualquier VPH desde el extremo inferior de edad, e incluso los casos con más de un genotipo de VPH a edad temprana, corrobora la recomendación de implementar métodos moleculares de tamizaje y de educar a la población en riesgo sobre salud sexual. 
De los casos VPH-positivos, el más frecuente fue VPH$16(23,8 \%)$, cifra superior a lo reportado por Santos quien encontró una frecuencia de $16 \%{ }^{(5)}$. No se pudo definir el genotipo de VPH del $38,4 \%$ de los casos debido a características de la metodología utilizada en el presente estudio. El uso de otras enzimas de restricción como Hae III, Dd I, Pst I, Hinf I, Bam HI Sau 3AI, o secuenciamiento génico del VPH podría permitirnos identificar otros genotipos de alto riesgo no reconocidos por AFA1, como el VPH-52 que estuvo presente en el $3,2 \%$ de las mujeres sanas del trabajo de Santos et al. ${ }^{(5)}$. En la actualidad, se ha incrementado el número de enzimas de restricción al análisis de tamizaje que se realiza en el INEN.

Los casos catalogados como VPH-negativos estudiados con cebadores específicos MY09/MY11 para el gen L1, deberían de ser corroborados mediante la amplificación de otros genes como E6 y E7 para completar el estudio y descartar la posibilidad de algún falso negativo, como en el estudio realizado por Santos quien encontró que 19 de las 29 muestras negativas fueron realmente positivas al estudiarse el oncogén E7 ${ }^{(5)}$.

La edad y el estado civil sirven como importantes marcadores de riesgo de exposición de VPH y otras infecciones de transmisión sexual. Ser soltera se asoció con la presencia de infección de VPH-AR. En forma similar a lo reportado por investigadores de otros países, y probablemente relacionado con ausencia de educación sobre salud sexual y métodos de protección contra enfermedades de transmisión sexual. El largo intervalo entre la infección por VPH y el desarrollo del cáncer explicaría por qué el CCU es más frecuente en mujeres mayores y casadas ${ }^{(10)}$.

Una limitante del presente estudio es la ausencia de información de otros factores asociados con la presencia de VPH encontrados en otros estudios como edad de inicio de vida sexual y número de parejas sexuales, así como la ausencia de informes del estudio de citología (PAP) de las pacientes. El presente estudio tampoco hace el seguimiento clínico o mediante citología (PAP) de los casos $\mathrm{PVH}$-positivos con la finalidad de determinar el riesgo de desarrollar CCU. Finalmente, tuvimos la limitante que la información sociodemográfica de domicilio, grado de instrucción y estado civil fue recogida del sistema RENIEC y no se logró corroborar a través de contacto directo con los usuarios.

En conclusión, el $32,5 \%$ de los casos fueron VPH positivos, siendo en el genotipo de AR más frecuente el VPH 16 y el genotipo de BR el VPH 6. El grupo de mujeres de 17 a 29 años, y de estado civil soltera presentaron mayor frecuencia de casos VPH-positivos, por lo que se debería enfatizar su participación en programas de tamizaje con métodos moleculares y citológicos combinados como parte de un adecuado seguimiento a fin de detectar oportunamente a las mujeres en riesgo de desarrollar CCU y disminuir la morbimortalidad por cáncer en Perú.

Contribuciones de autoría: YSA, MDCCM, CAC y RMF participaron en la concepción y diseño del artículo; YSA, MCM, CAC, RMF, MC y EP participaron en la recolección /obtención de resultados, YSA, MDCCM, CAC, RMF y EP participaron en el análisis e interpretación de datos. YSA, MDCCM, CAC, MCG y RMF participaron en la redacción del artículo; YSA, MDCCM, CAC y RMF participaron en la revisión crítica del artículo; YSA, MDCCM, CAC y RMF realizaron la aprobación de su versión final; KDC participó en la asesoría estadística.

Fuentes de financiamiento: autofinanciado.

Conflictos de interés: los autores declaran no tener conflictos de interés.

\section{REFERENCIAS BIBLIOGRÁFICAS}

1. World Health Organization, International Agency for Research on Cancer. GLOBOCAN 2012: Estimated Cervical Cancer Mortality Worldwide in 2012 [Internet]. Washington DC: IARC; 2012 [citado el 22 de noviembre del 2013]. Disponible en: http://globocan.iarc.fr/ Pages/fact_sheets_cancer.aspx

2. Muñoz N, Bosch FX, de Sanjosé S, Herrero R, Castellsagué X, Shah KV, et al. Epidemiologic classification of human papillomavirus types associated with cervical cancer. N Engl J Med. 2003;348(6):518-27.

3. World Health Organization, International Agency for Research on
Cancer. Vol 100 B, a review of human carcinogens. Part B: biological agents [Internet]. Washington DC: IARC; 2012 [citado el 22 de noviembre del 2013]. Disponible en: http:// monographs.iarc.fr/ENG/Monographs/ vol100B/mono100B-1.pdf

4. Serrano B, Alemany L, Tous S, Bruni L, Clifford GM, Weiss T, et al. Potential impact of a nine-valent vaccine in human papillomavirus related cervical disease. Infect Agent Cancer. 2012;7(1):38. doi: 10.1186/17509378-7-38.

5. Santos C, Muñoz N, Klug S, Almonte M, Guerrero I, Alvarez M, et al.
HPV types and cofactors causing cervical cancer in Peru. Br J Cancer. 2001;85(7):966-71.

6. Young LS, Bevan IS, Johnson MA, Blomfield PI, Bromidge T, Maitland NJ, et al. The polymerase chain reaction: a new epidemiological tool for investigating cervical human papillomavirus infection. BMJ. 1989;298(6665):14-8.

7. Mandelblatt JS, Lawrence WF, Womack SM, Jacobson D, Yi B, Hwang YT, et al. Benefits and costs of using HPV testing to screen for cervical cancer. JAMA. 2002;287(18):2372-81. 
8. Almonte M, Ferreccio C, Winkler JL, Cuzick J, Tsu V, Robles S, et al. Cervical screening by visual inspection, HPV testing, liquid-based and conventional cytology in Amazonian Peru. Int J Cancer. 2007;121(4):796-802.

9. Freeman H, Wingrove B. Excess cervical cancer mortality: a marker for low access to health care in poor communities [Internet]. Maryland: National Cancer Institute; 2005. [citado el 11 de Mayo del 2015]. Disponible en: http://www.cancer. gov/about-nci/organization/crchd/ about-health-disparities/resources

10. Kahn JA, Lan D, Kahn RS. Sociodemographic factors associated with high-risk human papillomavirus infection. Obstet Gynecol. 2007;110(1):87-95.

11. de Roda Husman AM, Walboomers JM, Meijer CJ, Risse EK, Schipper ME, Helmerhorst TM, et al. Analysis of cytomorphologically abnormal cervical scrapes for the presence of 27 mucosotropic human papillomavirus genotypes, using polymerase chain reaction. Int J Cancer. 1994;56(6):802-6.

12. Jacobs MV, de Roda Husman AM, van den Brule AJ, Snijders PJ, Meijer CJ, Walboomers JM. Group-specific differentiation between high- and lowrisk human papillomavirus genotypes by general primer-mediated PCR and two cocktails of oligonucleotide probes. J Clin Microbiol. 1995;33(4):901-5.

13. Dybikowska A, Licznerski P, Podhajska A. HPV detection in cervical cancer patients in northern Poland. Oncol Rep. 2002;9(4):871-4.

14. Iwasaki R, Galvez-Philpott F, AriasStella J Jr, Arias-Stella J. Prevalence of high-risk human papillomavirus by cobas $4800 \mathrm{HPV}$ test in urban Peru. Braz J Infect Dis. 2014;18(5):469-72. doi: 10.1016/j.bjid.2014.01.010.

15. Li Ning ML, Bazan M, Arias-Stella JJ. HPV DNA testing in apopulation with high prevalence of cervical squamous carcinoma: 5-year experience in urban Peru. Denver, CO: USCAP: United States \& Canadian Academy of Pathology; 2008. p. 1-7.

16. Cervantes J, Lema C, Hurtado L, Andrade R, Quiroga G, Garcia G, et al. Prevalence of human papillomavirus infection in rural villages of the Bolivian Amazon. Rev Inst Med Trop S Paulo. 2003;45(3):131-5.

17. Camara GN, Cerqueira DM, Oliveira AP, Silva EO, Carvalho LG, Martins CR. Prevalence of human papillomavirus types in women with pre-neoplastic and neoplastic cervical lesions in the Federal District of Brazil. Mem Inst Oswaldo Cruz. 2003;98(7):879-83.

18. Ferreccio C, Prado RB, Luzoro AV, Ampuero SL, Snijders PJ, Meijer CJ, et al. Population-based prevalence and age distribution of human papillomavirus among women in Santiago, Chile. Cancer Epidemiol Biomarkers Prev. 2004;13(12):2271-6.

19. Molano M, Posso H, Weiderpass E, van den Brule AJ, Ronderos M, Franceschi $S$, et al. Prevalence and determinants of HPV infection among Colombian women with normal cytology. $\mathrm{Br} \mathrm{J}$ Cancer. 2002;87(3):324-33.

20. Lazcano-Ponce E, Herrero R, Muñoz N, Cruz A, Shah KV, Alonso P, et al. Epidemiology of HPV infection among Mexican women with normal cervical cytology. Int $\mathrm{J}$ Cancer. 2001;91(3):412-20.

Corresponsal: Yasser Sullcahuaman Allende Dirección: Av. Angamos Este 2520-Surquillo, Lima-Perú

Teléfono: (511) 992157220

Correo electrónico:ysullcahuaman@gmail.com

REVISTA PERUANA DE MEDICINA EXPERIMENTAL Y SALUD PÚBLICA CUMPLIENDO SUS METAS Y PROYECTÁNDOSE AL FUTURO

\section{Visite los contenidos de la revista en: www.ins.gob.pe/rpmesp}

